\title{
Commentary A role for prophylactic antibiotics in necrotizing pancreatitis?
Why we may never know the answer ...
} Jan J De Waele

Department of Critical Care Medicine, Ghent University Hospital, De Pintelaan 185, 9000 Ghent, Belgium

Corresponding author: Jan J De Waele, jan.dewaele@ugent.be

See related research by Fritz et al., http://ccforum.com/content/12/6/R141

Published: 2 December 2008

This article is online at http://ccforum.com/content/12/6/195

(c) 2008 BioMed Central Ltd

\begin{abstract}
The use of prophylactic antibiotics in patients with severe acute pancreatitis remains an intensely debated topic. Although animal studies consistently demonstrated an advantage of antibiotic prophylaxis, the only two blinded randomized controlled trials could not confirm these findings. Translation of the experimental models in human clinical practice is hampered by a number of fundamental differences between experimental pancreatitis and human disease, and therefore it is highly unlikely that the pronounced benefit found in experimental pancreatitis will ever be demonstrated in human disease. Early and accurate risk stratification to identify the patient at risk for infection early in the course of the disease seems to be the greatest challenge. Until we are able to demonstrate an advantage of antibiotic prophylaxis in a high-risk human population, the absence of proven benefit and potential side effects of this strategy should be acknowledged and the use of antibiotics should be limited to the treatment of documented infection.
\end{abstract}

\section{Early therapeutic or prophylactic administration of antibiotics?}

In this issue of Critical Care, Fritz and colleagues [1] describe how, in a rat model of necrotizing pancreatitis, prophylactic administration of antibiotics reduced mortality at 24 hours after induction of pancreatitis from $57 \%$ to $0 \%$. Furthermore, they compared early antibiotic treatment - antibiotics started 24 hours after induction of pancreatitis (that is, the time frame in which infection of necrosis is expected to be present) - with prophylactic antibiotic administration and found that early antibiotic treatment reduced pancreatic superinfection, but to a lesser extent than prophylactic administration of antibiotics. Parallel to this observation, they found that mortality is again significantly lower after prophylactic antibiotics; animals receiving early antibiotic treatment had a lower mortality rate compared with controls, but the difference was not statistically significant $(27 \%$ versus 43\%).
The originality of this study lies in the 'early antibiotic treatment' arm, in which the investigators aimed to simulate the clinical situation in which antibiotics are started upon diagnosis of established infection. Previous animal research focused mainly on antibiotic prophylaxis versus placebo, with an advantage for the intervention [2,3]. Fritz and colleagues demonstrated that, compared with therapeutic administration of antibiotics, prophylactic antibiotics decreased the rate of pancreatic superinfection. This has been studied before, and similar findings were reported by Cinar and colleagues [4]. But in a study by Schwarz and colleagues [5], there was no difference between prophylactic and early therapeutic administration of both meropenem and ciprofloxacin in an animal model.

\section{How to translate these findings in clinical practice}

Although this experimental study suggests an advantage of prophylactic administration compared with early therapeutic administration of antibiotics in rats with severe acute pancreatitis, it is highly questionable whether the results observed in this experimental setting can be easily translated to clinical practice. Several of the arguments advocated by Fritz and colleagues to explain why randomized controlled trials on antibiotic prophylaxis in humans did not provide conclusive results are the same arguments that explain why the results of animal experiments are of limited use for the clinician. First of all, in this animal model, a standard procedure at a predefined moment induces acute pancreatitis with similar dynamics in all animals, with extensive necrosis as a result. In human acute pancreatitis, the situation is completely different: patients with pancreatitis present at different moments after the start of symptoms, and thus at different stages of the pancreatitis, with different causes. Some present early with only mild edema, whereas 
others will present later when necrosis (with variable extent) or even superinfection has already been established. Therefore, prophylactic administration of antibiotics before necrosis is present - if at all desirable - is often not possible in clinical practice. Second, in this animal model, the exact moment of bacterial superinfection is well known: within the first 8 to 24 hours, the necrosis is infected in all animals, but in human pancreatitis, infection may occur within the first days but has its highest incidence after 7 to 21 days. In experimental pancreatitis, it is therefore possible to give antibiotics effectively, without the risk of developing resistance due to prolonged exposure to antibiotics or the risk of selection of microorganisms as observed in human pancreatitis, which has been documented both in retrospective studies [6] as well as the randomized controlled trials on this subject $[7,8]$. Third, in the animal model of pancreatitis, all animals develop pancreatic superinfection, whereas in human pancreatitis, prediction of who will develop infection remains one of the most challenging issues in the management of these patients [9]. The majority of patients will not develop infected pancreatic necrosis and there are no reliable tools available for early (that is, within the first days after the start of symptoms) identification of patients at the highest risk. Finally, the effects of other (new) interventions often used in human pancreatitis such as support of organ dysfunction, the use of enteral nutrition, and so on are not evaluated in experimental pancreatitis. These factors very likely will decrease bacterial translocation, which is considered to be the mechanism that leads to bacterial superinfection, and therefore may affect the risk for pancreatic infection.

\section{Time for a change}

For all of the above reasons, the beneficial effect of prophylactic antibiotics in the ideal standardized setting of experimental necrotizing pancreatitis has not been reproduced convincingly in any human study, and very likely, this will never be the case. The reason for this is that acute pancreatitis is a complex disease with a variable presentation and a highly unpredictable course, and we are not able to identify - early in the course of the disease - the patient who is at the highest risk for infection. This necessitates the inclusion in clinical studies of a lot of patients who will never develop infection; in the past, this has led to a lower-than-predicted incidence of infection, with resulting underpowered studies.

A potential explanation for this is the current array of dogmas used in severity prediction. We should acknowledge that there are two major complications in pancreatitis, with a bimodal distribution of mortality: early organ dysfunction and late infectious complications. As long as we continue to define severe disease as either one of these [10] (among other complications that qualify for the category 'severe disease') and as long as we do not realize that these two major complications are entirely distinct entities with different risk profiles, we may not be able to improve the accuracy of our risk stratification tools.

\section{Role of antibiotics in severe acute pancreatitis}

Until we are able to identify the patient truly at risk for infection and have a treatment modality that has no relevant side effects and that can be initiated in a timely fashion, the role of antibiotics in patients with established pancreatitis remains in the treatment of documented infection. When suspected, infection should be actively sought, and computed tomography-guided fine-needle aspiration of the suspected area should be performed. Timely initiation of antibiotics is essential in pancreatic infection, just as in any other (intra-abdominal) infection. Therefore, a low threshold for an aggressive diagnostic approach to 'search for and destroy' infection is still warranted.

\section{Competing interests}

The author declares that he has no competing interests.

\section{References}

1. Fritz S, Hartwig W, Lehmann R, Will-Schweiger K, Kommerell M, Hackert T, Schneider L, Buchler M, Werner J: Prophylactic antibiotic treatment is superior to therapy on-demand in experimental necrotizing pancreatitis. Crit Care 2008, 12: R141.

2. Mithofer K, Fernandez-del Castillo C, Ferraro MJ, Lewandrowski K, Rattner DW, Warshaw AL: Antibiotic treatment improves survival in experimental acute necrotizing pancreatitis. Gastroenterology 1996, 110:232-240.

3. Foitzik T, Klar E, Buhr HJ, Herfarth $\mathrm{C}$ : Improved survival in acute necrotizing pancreatitis despite limiting the indications for surgical debridement. Eur J Surg 1995, 161:187-192.

4. Cinar E, Ateskan U, Baysan A, Mas MR, Comert B, Yasar M, Ozyurt M, Yener N, Mas N, Ozkomur E, Altinatmaz K: Is late antibiotic prophylaxis effective in the prevention of secondary pancreatic infection? Pancreatology 2003, 3:383-388.

5. Schwarz M, Poch B, Isenmann R, Kriese D, Rozdzinski E, Beger $H G$, Gansauge F: Effect of early and late antibiotic treatment in experimental acute pancreatitis in rats. Langenbecks Arch Surg 2007, 392:365-370.

6. De Waele JJ, Vogelaers D, Hoste E, Blot S, Colardyn F: Emergence of antibiotic resistance in infected pancreatic necrosis. Arch Surg 2004, 139:1371-1375.

7. Dellinger EP, Tellado JM, Soto NE, Ashley SW, Barie PS, Dugernier T, Imrie CW, Johnson CD, Knaebel HP, Laterre PF, Maravi-Poma E, Kissler JJ, Sanchez-Garcia M, Utzolino S: Early antibiotic treatment for severe acute necrotizing pancreatitis: a randomized, double-blind, placebo-controlled study. Ann Surg 2007, 245:674-683.

8. Isenmann R, Runzi M, Kron M, Kahl S, Kraus D, Jung N, Maier L, Malfertheiner P, Goebell H, Beger HG: Prophylactic antibiotic treatment in patients with predicted severe acute pancreatitis: a placebo-controlled, double-blind trial. Gastroenterology 2004, 126:997-1004.

9. De Waele JJ: Clinical research in acute pancreatitis and the failure to predict severe disease. Ann Surg 2007, 246:689.

10. Bradley EL 3rd: A clinically based classification system for acute pancreatitis. Summary of the International Symposium on Acute Pancreatitis, Atlanta, Ga, September 11 through 13, 1992. Arch Surg 1993, 128:586-590. 